\title{
Trend Analysis for the Choice and Cost of Lung Cancer Treatment in South Korea, 2003-2013
}

\author{
Dohun Kim, MD, PhD \\ So Young Kim, MD, PhD ${ }^{2,3}$ \\ Beomseok Suh, $\mathrm{MD}^{4}$ \\ Jong Hyock Park, MD, MPH, PhD
}

${ }^{1}$ Department of Thoracic and Cardiovascular Surgery, College of Medicine, Chungbuk National University and Chungbuk National University Hospital, Cheongju, ${ }^{2}$ Department of Public Health and Preventive Medicine, Chungbuk National University Hospital, Cheongiu, ${ }^{3}$ College of Medicine/Graduate School of Health Science Business Convergence, Chungbuk National University, Cheongju, ${ }^{4}$ Department of Family Medicine, Seoul National University Hospital, Seoul, Korea

Correspondence: Jong Hyock Park, MD, MPH, PhD College of Medicine/Graduate School of Health Science Business Convergence,

Chungbuk National University, 1 Chungdae-ro, Seowon-gu, Cheongju, 28644, Korea

Tel: 82-43-261-2873

Fax: 82-43-261-3459

E-mail: jonghyock@gmail.com

Received January 26, 2017

Accepted July 17, 2017

Published Online September 4, 2017

\begin{abstract}
Purpose
Our study aimed to report the annual changes in lung cancer statistics and analyze trends in sociodemographic, medical, and financial factors from 2003 to 2013 in the national database from the Korean National Health Insurance (KNHI).
\end{abstract}

\section{Materials and Methods}

Among 7,489 patients with code $\mathrm{C} 34$ in $\mathrm{KNHI}$ database, only lung cancer patients newly diagnosed after 2003 were included in the study population, for a total of 4,582 patients. Descriptive statistics were used to characterize treatment patterns and medical costs according to sociodemographic factors.

\section{Results}

Approximately $70 \%$ of subjects were male, and the mean age was 67 years. Around $46 \%$ of patients were over 70 years old, and $12 \%$ were over 80 years old. The medical costs were highest for patients younger than 60 and lowest for those over 80 years old. Surgery was more common in younger patients, while "no treatment" increased greatly with age. In trend analysis, the proportions of aging ( $p$ for trend $<0.001$ ), female ( $p$ for trend $=0.003$ ), metropolitan/urban ( $p$ for trend=0.041), and lowest or highest-income patients ( $p$ for trend $=0.004$ ) increased over time, along with the prevalence of surgery as the primary treatment ( $p$ for trend $<0.001$ ). There was also a trend with regard to change in medical costs ( $p$ for trend $<0.001$ ), in that those of surgery and radiotherapy increased.

\section{Conclusion}

Surgery as a curative treatment has increased over the past decade. However, the elderly, suburban/rural residents, and low-income patients were more likely to be untreated. Therefore, active measures are required for these increasingly vulnerable groups.

\section{Key words}

Lung neoplasms, Practice patterns, Pneumonectomy

\section{Introduction}

Lung cancer is the leading cause of death. In South Korea, lung surpassed stomach cancer as the leading cause of cancer death in 1999, and it is expected to account for $22.6 \%$ of all cancer deaths in 2012 [1]. Although the prevalence of lung cancer in males has decreased, it is still high among patients aged 65 and over [1]. In 2012, lung cancer killed an estimated 1,098,700 men and 491,200 women worldwide, correspon- ding to $24 \%$ and $14 \%$ of all cancer deaths in males and females, respectively [2].

Fortunately, lung cancer mortality has decreased due to smoking cessation, early diagnosis [3,4], and treatment improvements. Many countries, notably high-income ones, have seen a considerable decrease in the smoking prevalence for both males and females, so the trend of the lung cancer mortality is decreasing [5]. New surgical treatments have been adopted, such as video-assisted thoracic surgery, and have had good results, including earlier recovery, reduced 
Patients with code C34 claims in NHIS from 2002 to 2015 ( $n=7,489)$

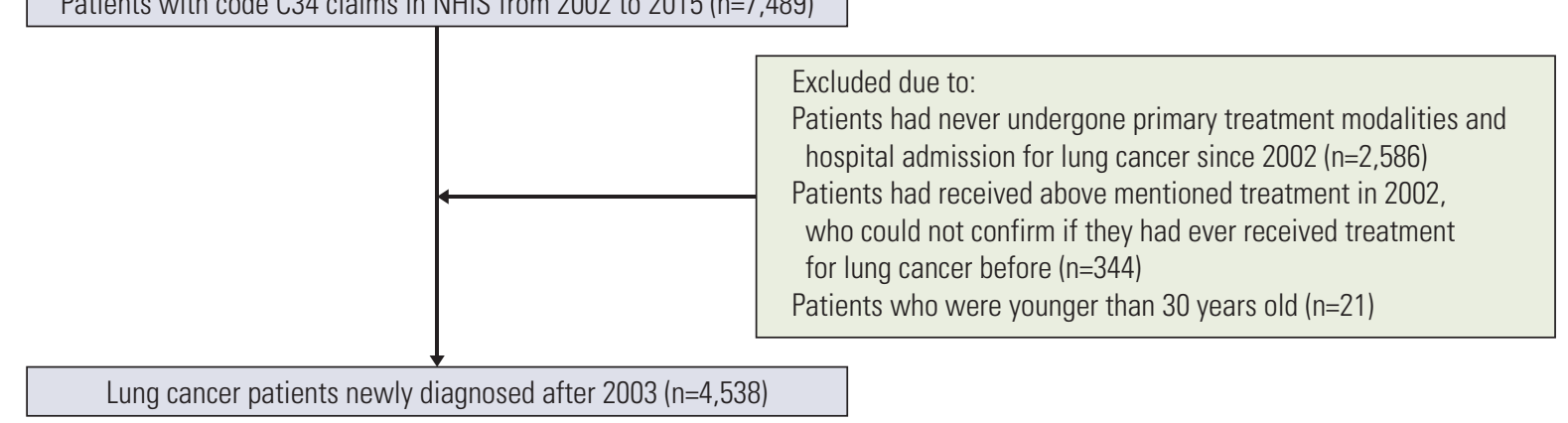

Fig. 1. Disposition of study subjects. NHIS, National Health Interview Survey.

chest pain, better cosmetics, and similar oncologic results $[6,7]$. Moreover, recent advances in systemic cytotoxic and molecularly targeted therapies have the potential to improve outcomes in lung cancer patients [8]. Advanced radiotherapy may be effective not only in the elderly, but also in younger cancer patients [9]. It is thought that the trends in the various factors related to lung cancer have resulted from the above improvements. Thus, it is important to understand these trends as a basis for creating efficient policies to overcome lung cancer and for effectively allocating resources. Trend data have already been examined in other countries and used to forecast changes in cancers [10,11]. However, such studies are lacking or have concentrated on certain areas in Korea $[12,13]$.

Therefore, the purpose of this study is to report the annual changes in lung cancer statistics in Korea, and to analyze the trends in sociodemographic, medical, and financial factors related to lung cancer from 2003 to 2013 with the national database from the Korean National Health Insurance (NHI).

\section{Materials and Methods}

\section{Data sources and subjects}

In South Korea, the NHI provides mandatory universal health insurance and offers comprehensive medical care coverage to all South Korean residents. Study data were obtained from 2002 to 2013 from the NHI Sharing Service provided by the NHI Corporation. These data were claims data from 1,000,000 people, representing $2 \%$ of the entire Korean population, randomly selected after the entire population was stratified according to sociodemographic param-
Table 1. Basic characteristics of study population

\begin{tabular}{|c|c|}
\hline Characteristic & No. $(\%)(n=4,538)$ \\
\hline \multicolumn{2}{|l|}{ Age (yr) } \\
\hline $30-49$ & $364(8.0)$ \\
\hline $50-59$ & 707 (15.6) \\
\hline $60-69$ & $1,403(30.9)$ \\
\hline $70-79$ & $1,504(33.2)$ \\
\hline$\geq 80$ & $560(12.3)$ \\
\hline \multicolumn{2}{|l|}{ Sex } \\
\hline Male & $3,210(70.7)$ \\
\hline Female & $1,328(29.3)$ \\
\hline \multicolumn{2}{|l|}{ Residence } \\
\hline Metropolitan & $791(17.4)$ \\
\hline Urban & $1,091(24.0)$ \\
\hline Suburban/Rural & $2,656(58.5)$ \\
\hline \multicolumn{2}{|l|}{ Income } \\
\hline 1st quartile & $866(19.1)$ \\
\hline 2nd quartile & 947 (20.9) \\
\hline 3rd quartile & $1,298(28.6)$ \\
\hline 4th quartile & $1,427(31.4)$ \\
\hline \multicolumn{2}{|c|}{ Primary treatment modality } \\
\hline OP & $891(19.6)$ \\
\hline RTx & $1,250(27.6)$ \\
\hline CTx & $1,991(43.9)$ \\
\hline \multicolumn{2}{|c|}{ Primary treatment regimen } \\
\hline OP only & $569(12.5)$ \\
\hline $\mathrm{OP}+\mathrm{CTx}$ & $202(4.4)$ \\
\hline $\mathrm{OP}+\mathrm{RTx}$ & $49(1.1)$ \\
\hline $\mathrm{OP}+\mathrm{CT} x+\mathrm{RT} x$ & $71(1.6)$ \\
\hline $\mathrm{CT} x+\mathrm{RT} x$ & $656(14.5)$ \\
\hline CTx only & $1,062(23.4)$ \\
\hline RTx only & $474(10.4)$ \\
\hline No Tx & $1,455(32.1)$ \\
\hline
\end{tabular}

OP, surgical treatment; RTx, radiation therapy; CTx, chemotherapy; Tx, treatment. 


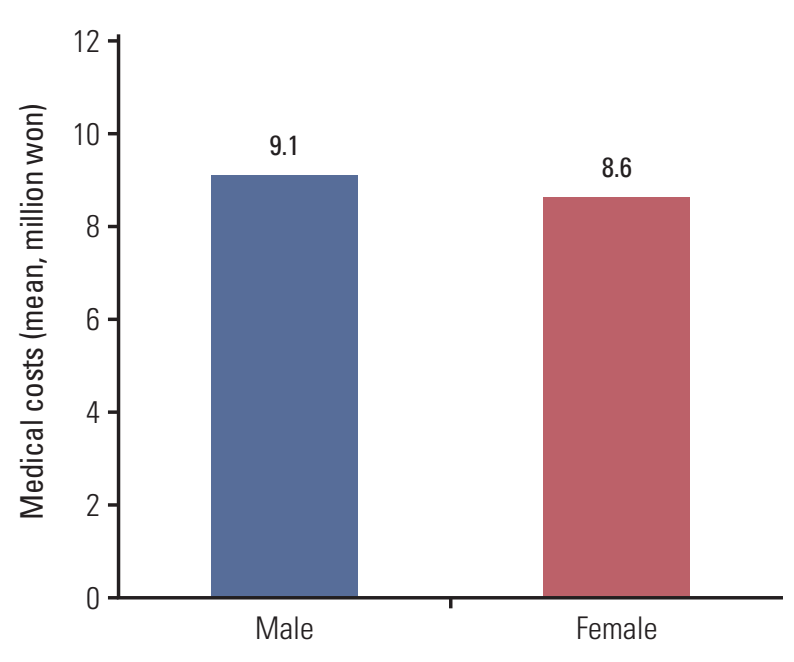

A
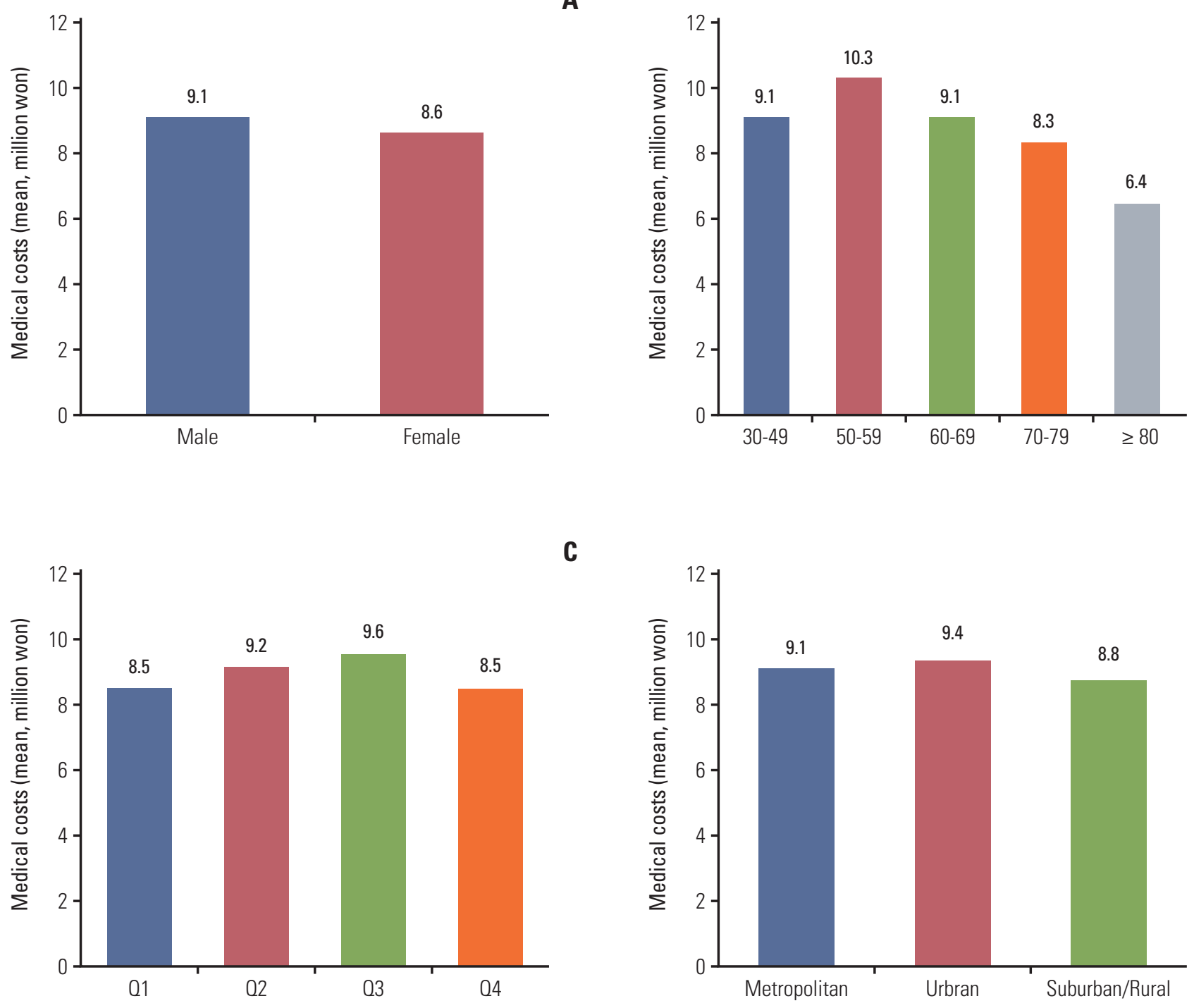

Fig. 2. Overview of medical costs and primary treatment regimens by sociodemographic factors of lung cancer patients in Korea, 2003-2013. Overview of the medical costs (in Korean won) of newly diagnosed lung cancer cases from 2003 to 2013 in Korea by sex (A), age group (B), income level (C), and residence (D) are shown. (Continued to the next page)

eters (age, sex, and income). The database includes detailed patient sociodemographic information, such as sex, age, health insurance premiums, residential area, comorbid diseases, treatment information including disease diagnoses, imaging and laboratory tests, procedures, and death outcomes. Patients with code C34 claims, indicating lung cancer according to the International Classification of Diseases, 10th edition, Clinical Modification (ICD-10-CM), were screened. Among 7,489 patients having code C34 from 2002 to 2013, only lung cancer patients newly diagnosed after 2003 were included, so that the confounding effects due to pre-diag- nosed lung cancer could be minimized. Early stage lung cancer can be billed as benign neoplasm at the time of surgery. Therefore, patients who were continuously billed as lung cancer (C34) after surgery were included in the study. Given that illegal billing practices such as upcoding occur in the healthcare industry, patients were also excluded if they only had a diagnosis of lung cancer, without histories of treatment and hospital admission $(n=2,586)$. Patients with code $C 34$ in $2002(n=344)$ and those younger than $30(n=21)$ were also excluded. Thus, a total of 4,538 patients with newly diagnosed lung cancer constituted the study population (Fig. 1). 


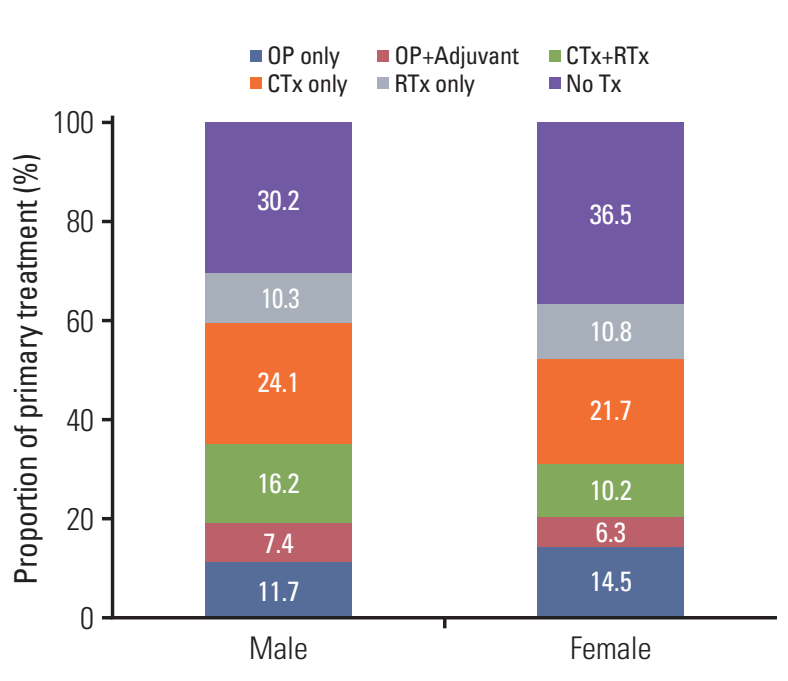

$\mathbf{E}$
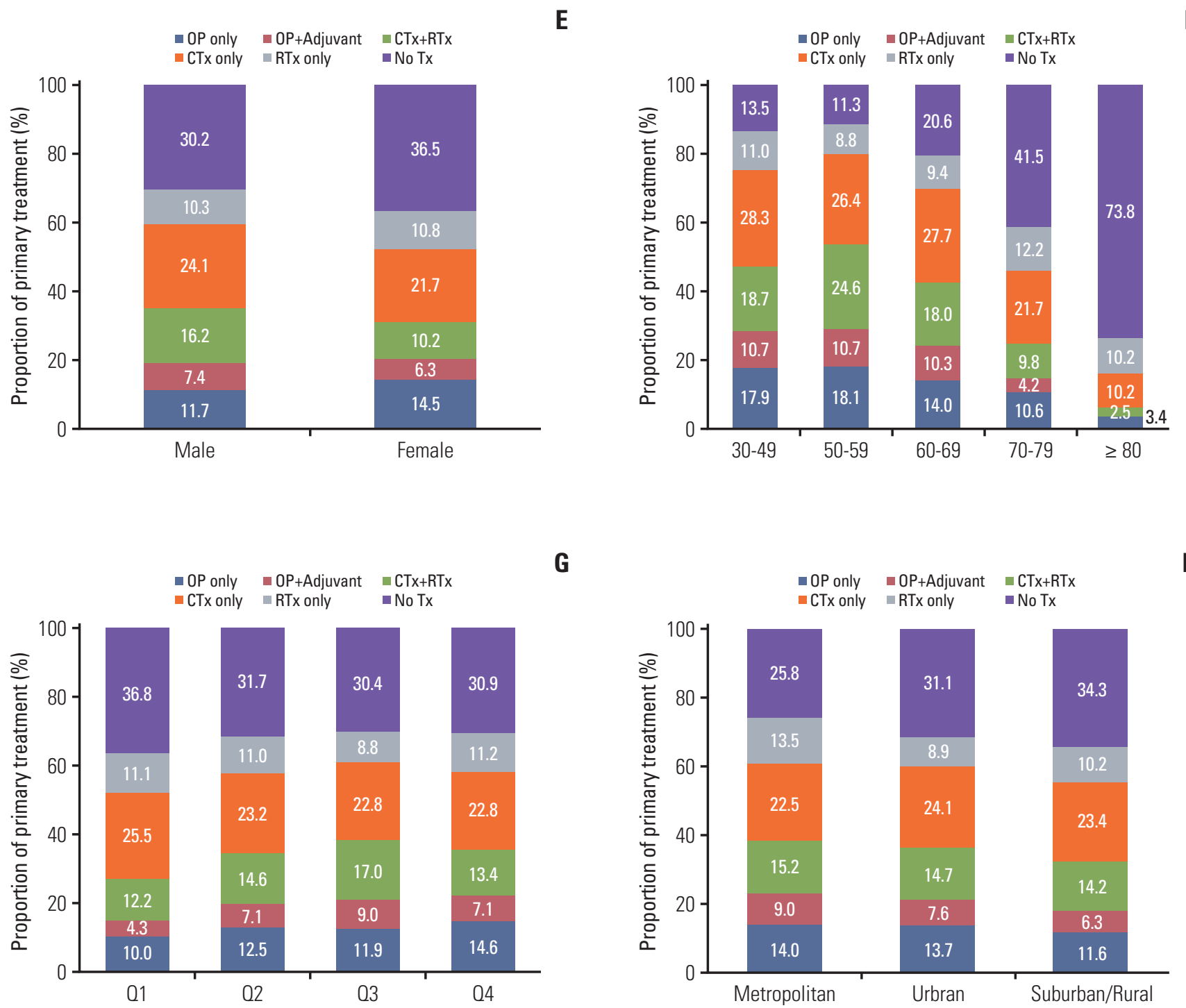

G



Fig. 2. (Continued from the previous page) The proportions of the primary treatment regimens by sex (E), age group (F), income level $(\mathrm{G})$, and residence $(\mathrm{H})$ are shown. OP, surgical treatment; RTx, radiation therapy; CTx, chemotherapy; OP+Adjuvant, $\mathrm{OP}+\mathrm{CT} x$ or $\mathrm{OP}+\mathrm{RT} x$ or $\mathrm{OP}+\mathrm{CTx}+\mathrm{RT} x ; \mathrm{Tx}$, treatment; $\mathrm{Q}$, quartile.

\section{Operational definition of primary treatment and costs}

We analyzed treatment patterns and medical costs in terms of the primary treatment modality, whether surgery (OP), radiation therapy $(\mathrm{RTx})$, or chemotherapy $(\mathrm{CTx})$. OP included open/thoracoscopic resection of the trachea or lungs, as well as mediastinal lymph node dissection (MLND) (Korean National Health Insurance [KNHI] reimbursement codes O1311-O1316, O1341-O1345, O1401-O1432, and O1596). The OP type was categorized as pneumonectomy, lobectomy, segmentectomy, wedge resection, and MLND only. The exploratory thoracotomy (KNHI reimbursement codes
'O1360') was not included. RTx encompassed all types of radiotherapy, including conformal and intensity-modulated RTx. Although patients may have undergone additional therapy before or after OP and RTx, they were categorized in terms of their primary treatment modality. CTx regimens used for lung cancer were applied in this study. The cost of each treatment modality was also calculated. "No treatment" was operationally defined as hospitalization with lung cancer (C34) without any treatment. 


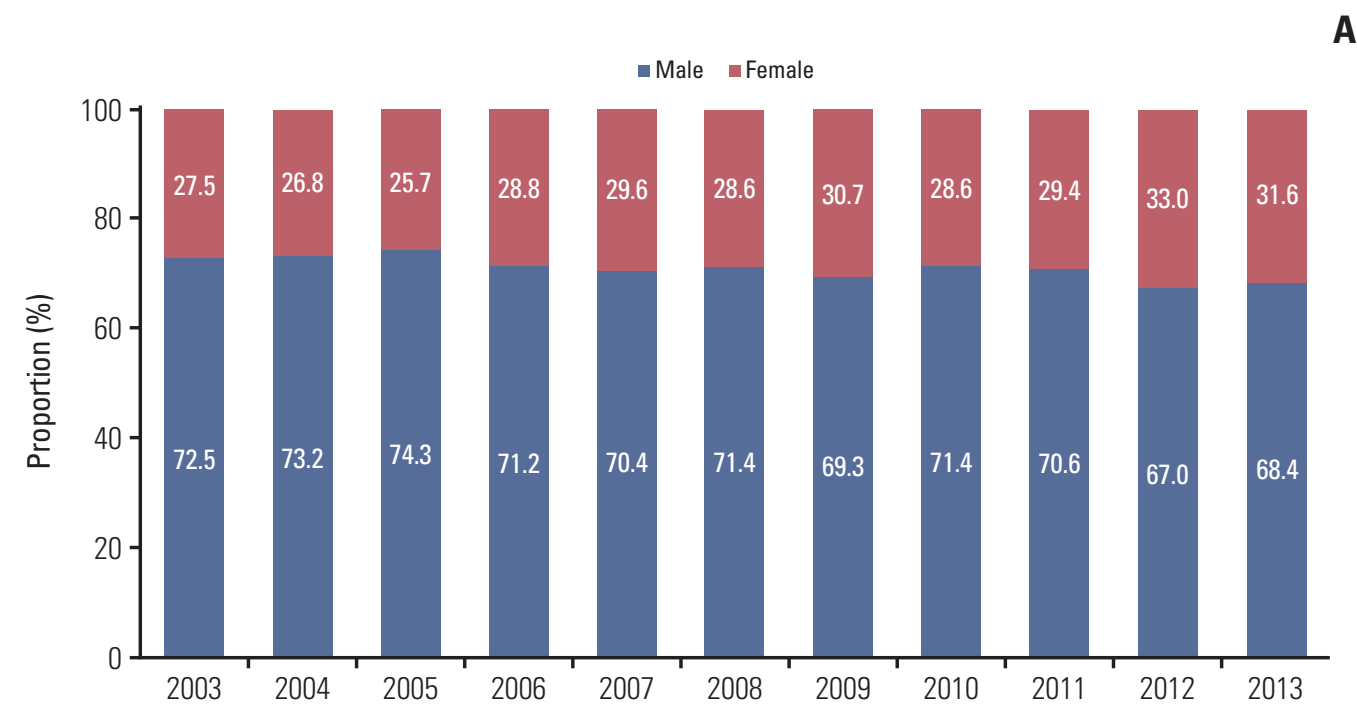

B

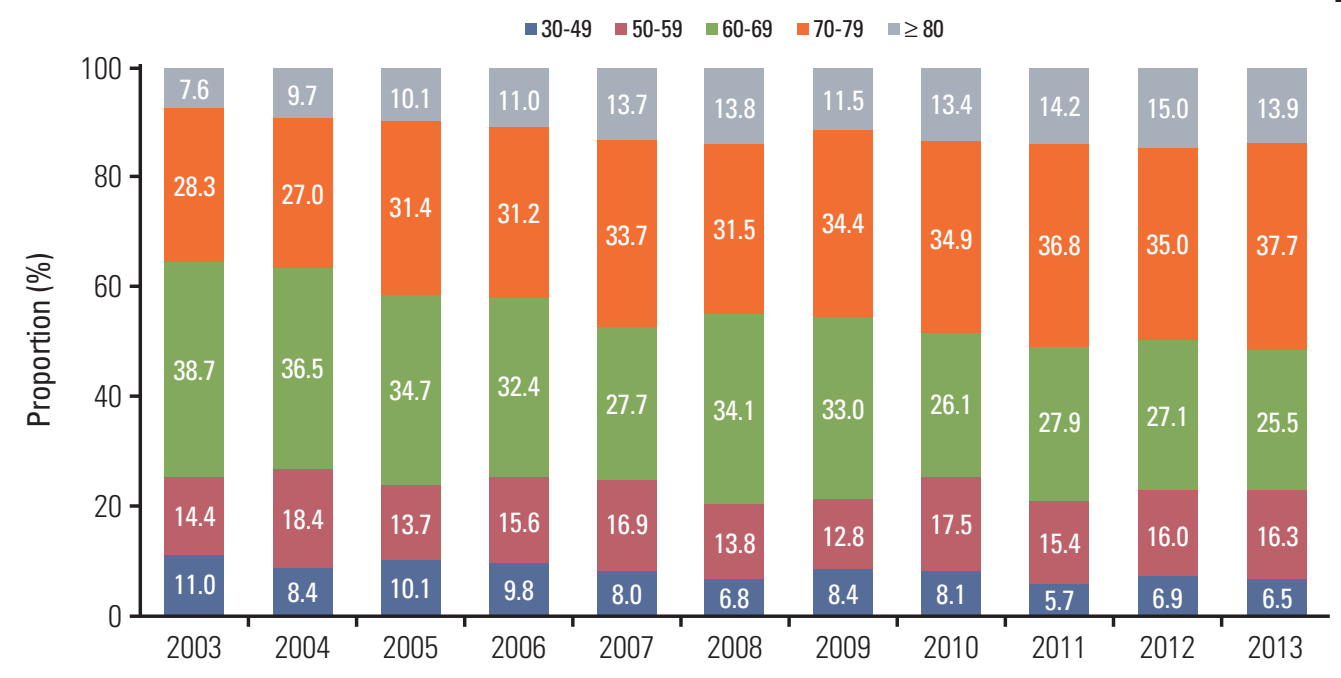

Fig. 3. Trends in lung cancer by sociodemographic factors in Korea, 2003-2013. Trends in newly diagnosed lung cancer cases by sociodemographic factors from 2003 to 2013 are shown based on the proportion of cases for each year. Trends by sex (A), age group (B), income levels (C), and residence (D) are shown. Q, quartile. (Continued to the next page)

\section{Variables and statistical analysis}

Patient sociodemographic parameters included age, income class, and residential area. Patients were divided into five categories according to their age at diagnosis $(<49,50$ $59,60-69,70-79$, and $\geq 80$ years). Income class was divided into quartiles as provided by the KNHI. Residential area was divided into three categories (metropolitan, urban, and suburban/rural) based on population density. Descriptive sta- tistics were used to characterize treatment patterns and medical costs according to sociodemographic factors. The annual $\mathrm{p}$ for trend was determined with a Wilcoxon type test for trend across ordered groups. All tests were two-tailed, with $\mathrm{p}<0.05$ considered significant. Statistical analyses were performed with Stata/SE software ver. 12.1 (Stata Corp., College Station, TX). 

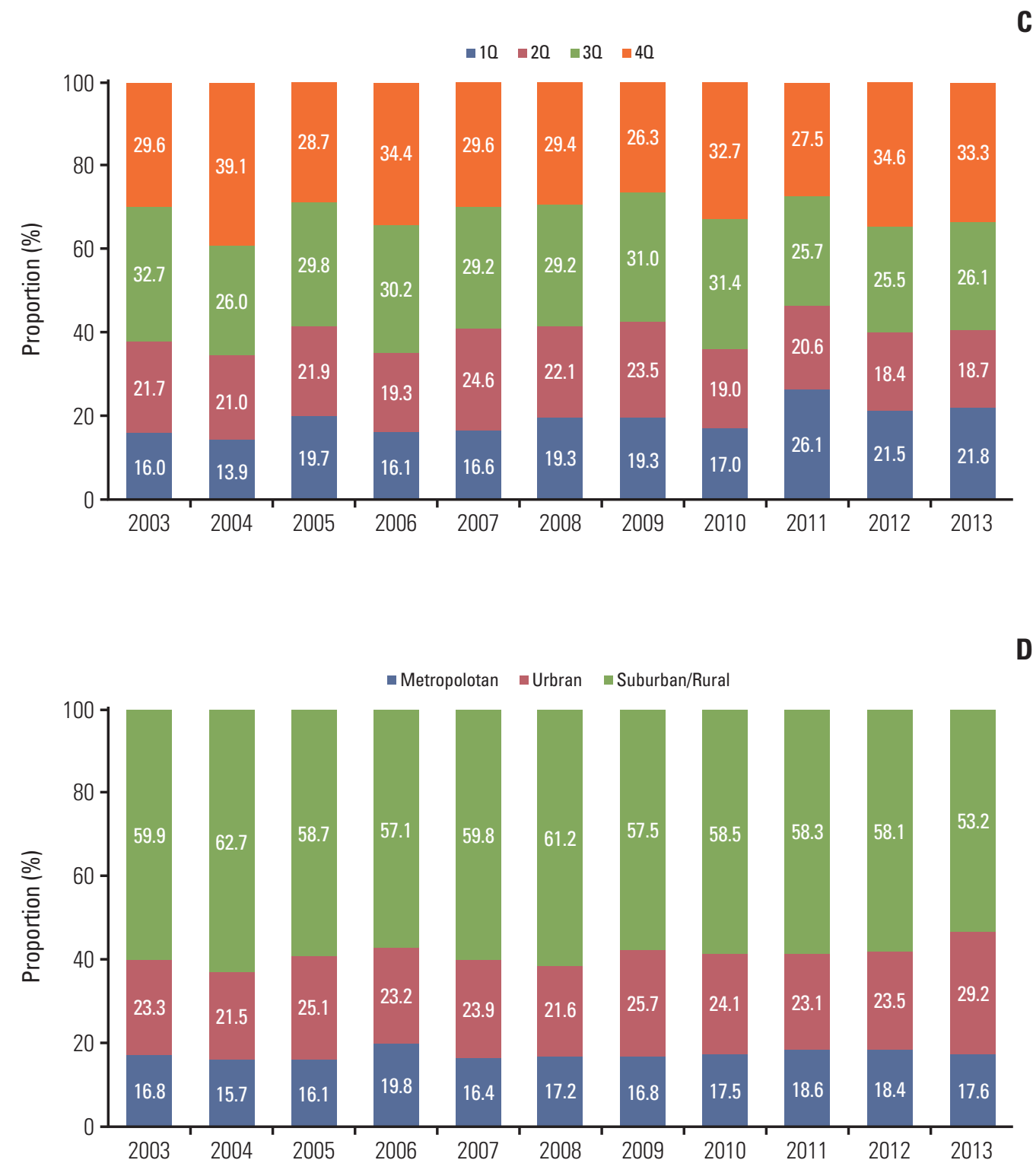

Fig. 3. (Continued from the previous page)

\section{Ethical statement}

The informed consent was waived and the study protocol was approved by the Institutional Review Board of Chungbuk National University (2015-04-005). 


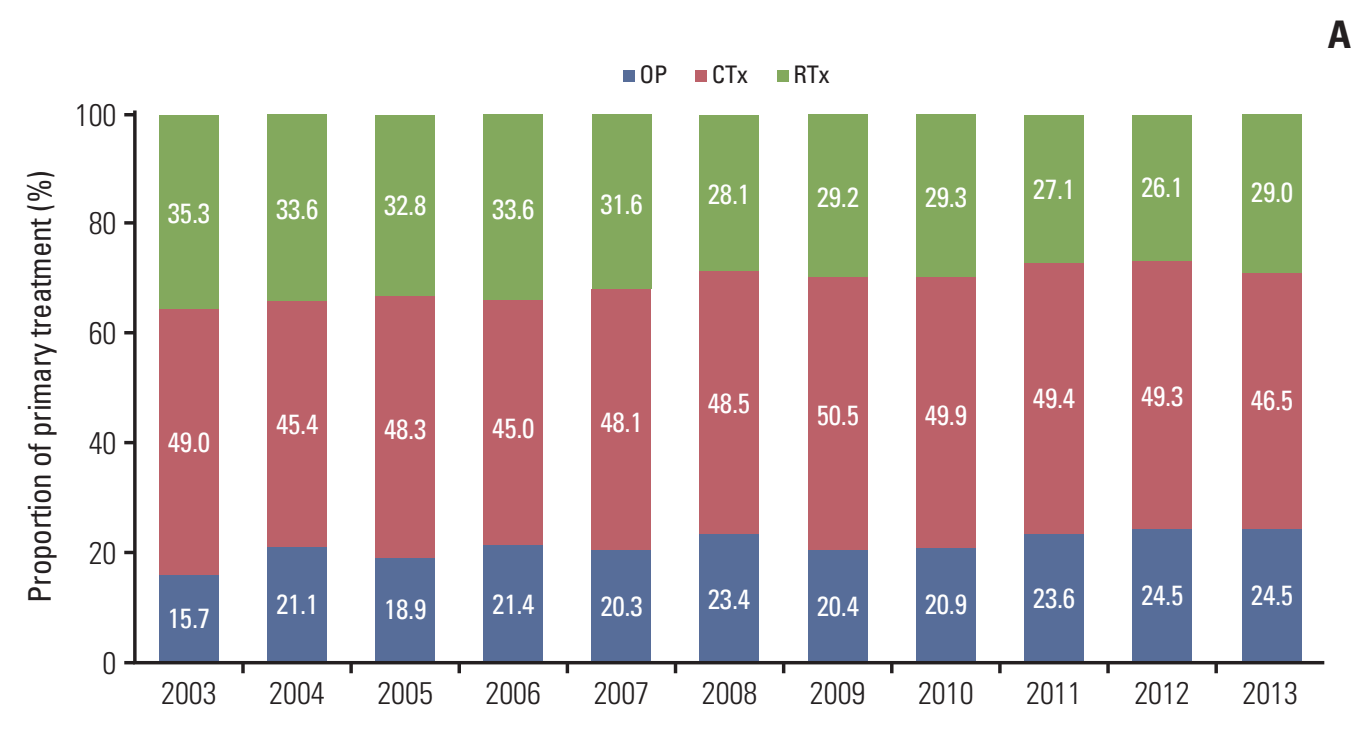

B

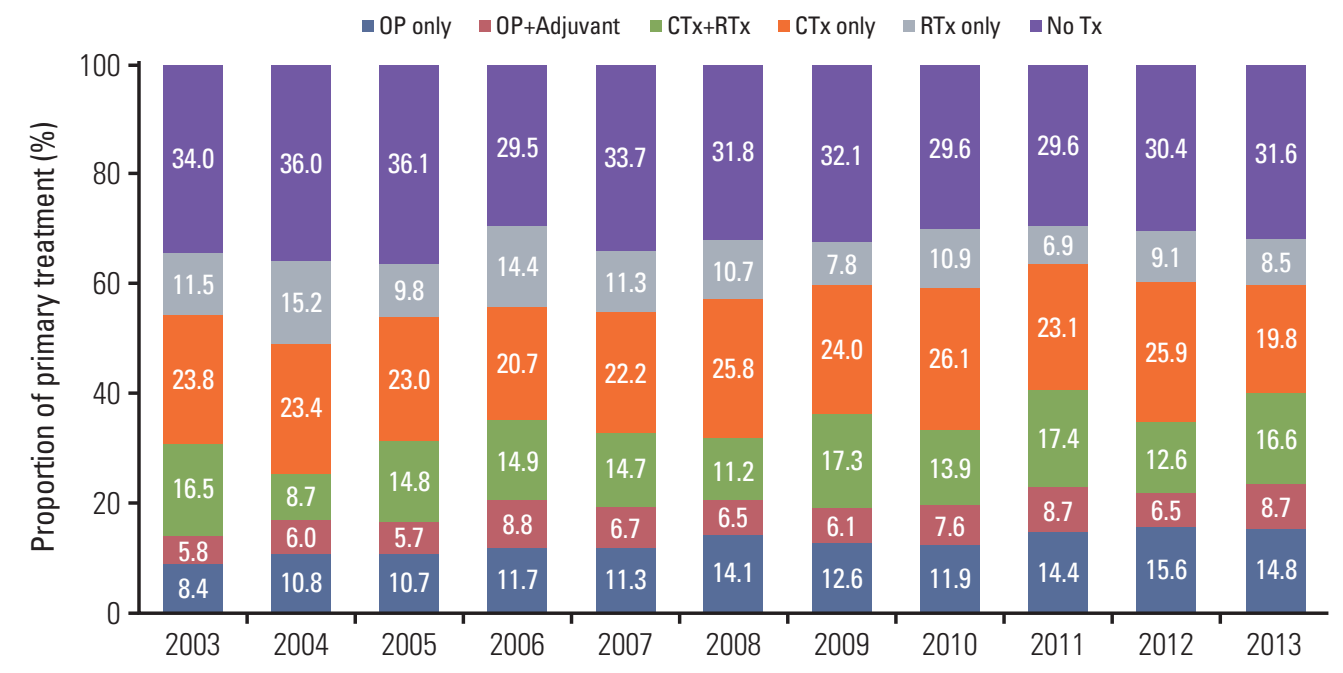

Fig. 4. Trends in the primary treatment of lung cancer in Korea, 2003-2013. Trends in the primary treatment of lung cancer in Korea from 2003 to 2013 are shown based on the proportion of newly diagnosed cases for each year. Trends in the primary treatment modality $(\mathrm{A})$, primary treatment regimen $(\mathrm{B})$, and primary surgical treatment $(\mathrm{C})$ are shown. $\mathrm{OP}$, surgical treatment; CTx, chemotherapy; RTx, radiation therapy; $\mathrm{OP}+$ Adjuvant, $\mathrm{OP}+\mathrm{CTx}$ or $\mathrm{OP}+\mathrm{RTx}$ or $\mathrm{OP}+\mathrm{CTx}+\mathrm{RTx}$; $\mathrm{Tx}$, treatment; MLND, mediastinal lymph node dissection. (Continued to the next page)

idence. Whereas income levels were generally evenly distributed, the fourth quartile contained the highest percentage of subjects $(31 \%)$. "No treatment" ("No Tx") was the most common primary treatment regimen, and "CTx only" was the next most common primary treatment regimen. Nineteen percent of subjects received OP as the primary treatment, while $26.7 \%$ received RTx and $43.9 \%$ received CTx.

\section{Medical costs and primary treatment regimens by sociodemographic factors}

The overall mean medical costs by sex, age, income, and residence are shown in Fig. 2A-D. The medical costs attributed to patients under the age of 60 were slightly higher than those attributed to other age groups (Fig. 2B), although the 


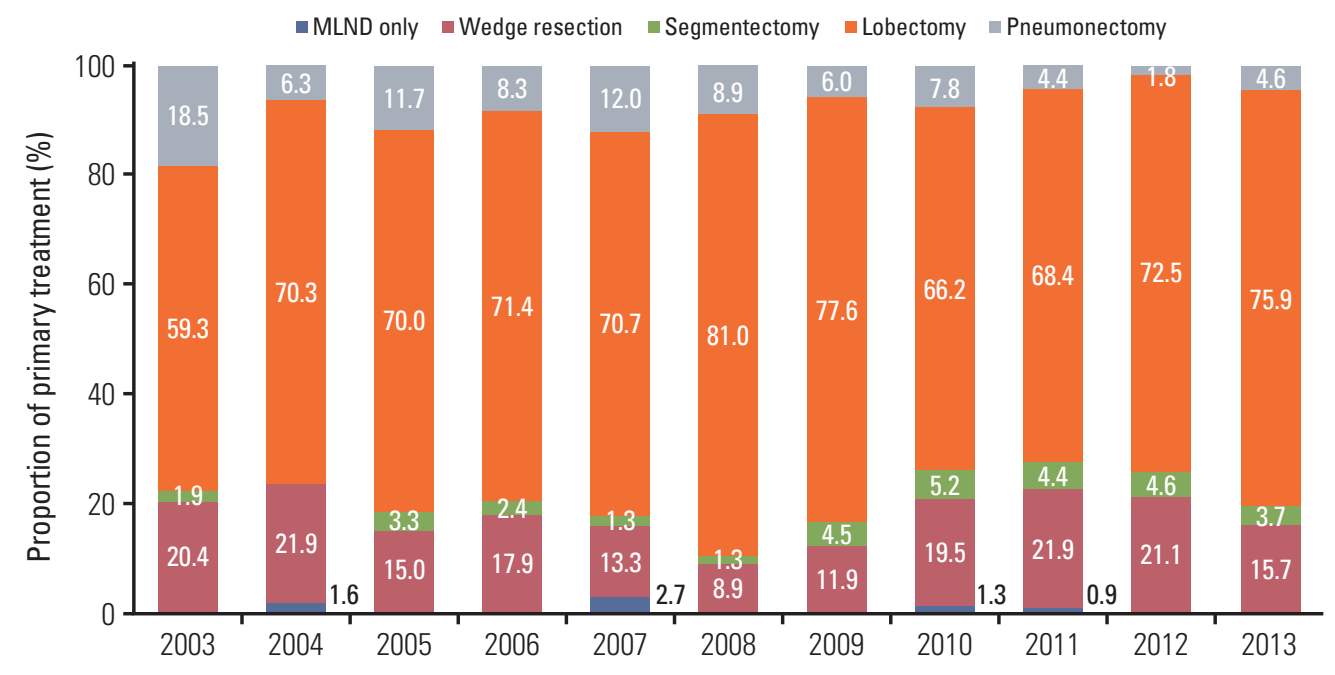

Fig. 4. (Continued from the previous page)

former made up only around $25 \%$ of the lung cancer cases during the 10-year study period (Fig. 3B). Similar trends were observed in residential areas and medical costs. The majority of lung cancer subjects came from suburban/rural areas (Fig. 3D), but the medical costs attributed to these subjects were markedly lower than those attributed to subjects from metropolitan or urban areas (Fig. 2D).

The distributions of primary treatment regimens according to various factors are shown in Fig. 2E-H. As expected, a markedly higher proportion of younger subjects received $\mathrm{OP}$, and "no treatment" highly increased with age. Notably, the proportion of subjects who received no treatment was much lower in metropolitan areas.

\section{Trends in lung cancer cases by sociodemographic factors}

There was a statistically significant trend of change in the gender distribution of lung cancer cases ( $p$ for trend $=0.003$ ), in which the proportion of females increased over the years (Fig. 3A). The age distribution also displayed a significant trend of change over time ( $\mathrm{p}$ for trend $<0.001$ ), as the proportions of subjects aged $70-79$ and $\geq 80$ increased, while the proportion of subjects aged 30-49 decreased (Fig. 3B). The overall proportion of lowest-income or highest-income group slightly increased over the years ( $\mathrm{p}$ for trend $=0.004$ ) (Fig. 3C). The residential area of lung cancer patients had a slight fluctuation over the years ( $p$ for trend=0.041) (Fig. 3D).

\section{Trends in primary treatment for lung cancer}

There was an overall increase in the proportion of subjects who received OP as the primary treatment ( $p$ for trend $<0.001$ ), whereas there was no overall significant change for RTx ( $p$ for trend=0.066) or CTx ( $p$ for trend=0.057) (Fig. 4A). There was a statistically significant trend of change in the primary treatment regimen over the years (overall $p$ for trend $<0.001$ ), where notably the proportion of subjects who received no treatment decreased, and the proportion of subjects who received only OP increased (Fig. 4B). There was not a statistically significant trend of change in the type of surgery ( $p$ for trend $=0.090$ ); nonetheless, the proportion of subjects who received pneumonectomy decreased, while the proportion of subjects who received lobectomy increased (Fig. 4C).

\section{Trends in medical costs for the primary treatment of lung cancer}

There was an overall statistically significant trend of change in the mean total medical cost for lung cancer treatment ( $\mathrm{p}$ for trend $<0.001$ ), notably for OP and RTx. The proportion of medical costs from CTx decreased (Fig. 5). 

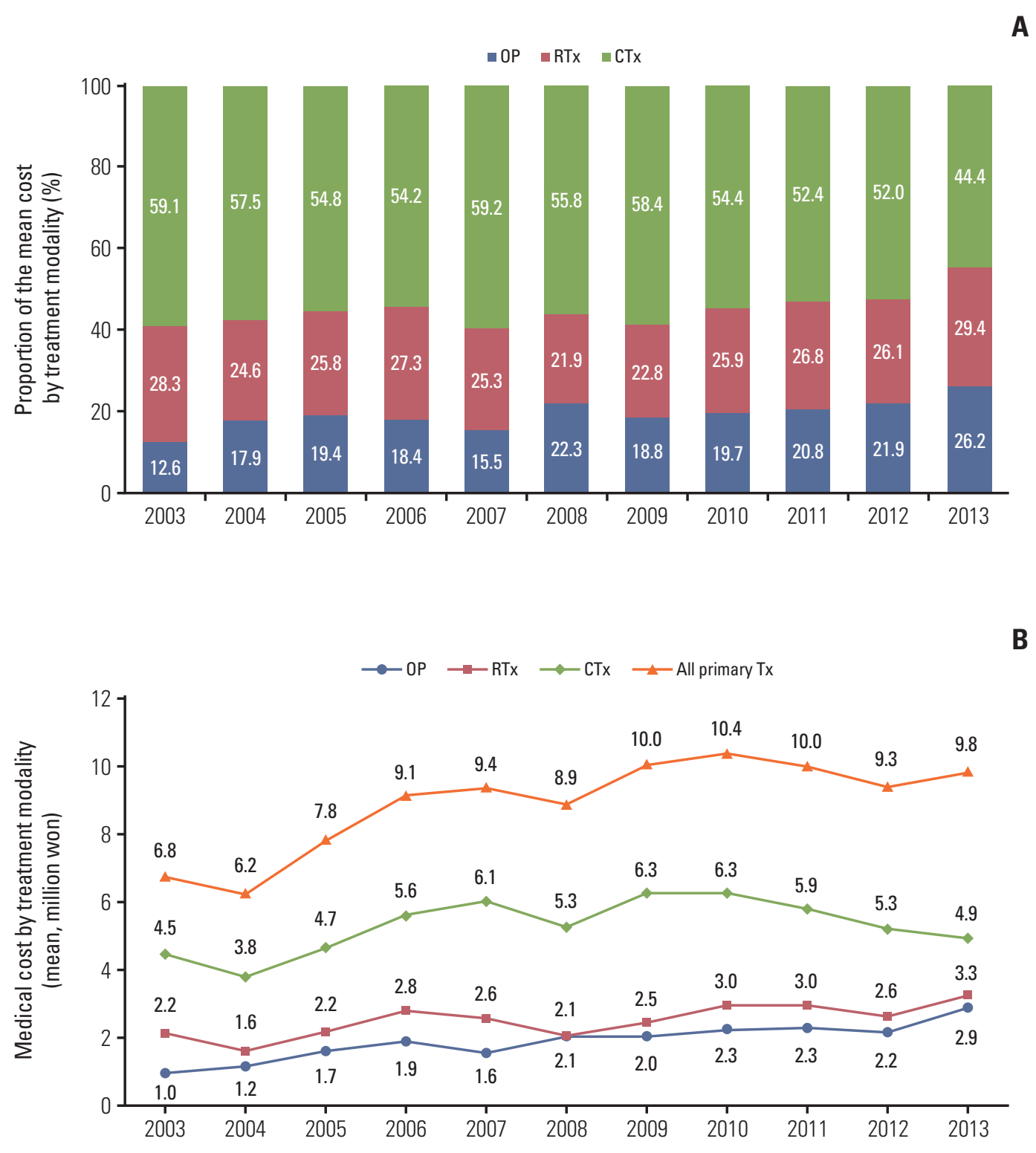

Fig. 5. Trends in medical costs for the primary treatment of lung cancer in Korea, 2003-2013. Trends in medical costs for the primary treatment of lung cancer in Korea from 2003 to 2013 are shown based on the mean cost for each primary treatment modality for each year. Trends are shown in terms of the proportion of the mean cost (A), as well as in terms of the absolute value of the mean cost in Korean won (B). OP, surgical treatment; RTx, radiation therapy; CTx, chemotherapy; Tx, treatment. $\mathrm{p}$ for trend, $\mathrm{OP}<0.0001, \mathrm{RT} x<0.001, \mathrm{CTx}=0.023$.

\section{Discussion}

Lung cancer was found to be a more prevalent malignancy in elderly, male, suburban/rural, and low-income subjects. Although lung cancer is becoming increasingly prevalent in aging individuals, there are also trends of increase in female, metropolitan/urban, and high-income patients. Female smokers are not rare, but are increasing in prevalence, especially among adolescents in Korea [14]. In addition, it is wellknown that traffic-related air pollution significantly increases the risk of lung cancer and can shorten the survival of lung cancer patients $[15,16]$. Therefore, it is understandable that the proportion of female and metropolitan/urban lung can- 
cer patients has increased in Korea. One reason for the increase in high-income patients may be that most of them tend to live in urban or metropolitan regions. Other factors should also be considered, including the greater exposure to air pollution and the lower daily activity levels than those of suburban/rural residents [15,17].

The prevalence of surgery increased during the study period, while the proportion of non-treated patients decreased. The increase in surgery may be due to the early detection of lung cancer and the extension of surgical indications [3,1820]. In particular, lobectomy (the standard treatment for lung cancer) has increased, rather than pneumonectomy (a relatively easy operation that nevertheless has many complications) [21]. Although the overall proportion of non-treated patients has decreased, more suburban/rural and elderly patients have given up treatment. In other words, the willingness to cure has become the lowest where the greatest number of patients has developed.

The medical cost of lung cancer was greatest in male, younger, and higher-income patients. Costs decreased with age, but this may not reflect the real demand for medical resources, because the proportion of non-treated patients also increased with age. It may be similar in residence (metropolitan/urban > suburban/rural). In trend analysis, medical costs increased gradually, notably for surgery and radiotherapy. This may be partly because of newly developed techniques or machines $[7,9,22]$. The medical cost of $\mathrm{Ctx}$ decreased, but CTx is still the mainstay of lung cancer treatment, and the cost may increase in the future as new medicines are developed, not only due to the price itself, but also due to the extension of indications [23].

There are some limitations to this study. First, we used NHI claims data of 1,000,000 cohort representing $2 \%$ of the entire Korean population. This database has sufficient representation, but the study subjects were relatively small.
Health insurance did not cover target agents such as bevacizumab, cetuximab, crizotinib during the study period. Therefore, the CTx costs may have been underestimated even though they were taken. Second, survival analysis was not possible due to the characteristics of the data (there was no exact TNM staging, which is a critical factor in the determination of survival). Further studies merging data from the Korean cancer registry will be helpful in providing such detailed information [24]. Third, it is difficult to envision this report being useful for years to come, because many new techniques, machines, and medicines will be adopted or are beginning to be adopted already. However, these data will help to guide policy decisions regarding lung cancer treatment and the distribution of medical resources, and are expected to be of value as a reference in the future.

In conclusion, lung cancer is characterized as affecting elderly, male, suburban, and low-income individuals, but is exhibiting new trends of acceleration among aging, female, metropolitan/urban, and high-income individuals. Surgery as a curative treatment has increased over the past decade. However, the elderly, suburban/rural residents, and lowincome patients were more likely to be untreated. Therefore, active measures are required for these increasingly vulnerable groups.

\section{Conflicts of Interest}

Conflict of interest relevant to this article was not reported.

\section{Acknowledgments}

This work was supported by the research grant of the Chungbuk National University in 2015 and National Research Foundation of Korea(NRF) grant funded by the Korea government(MSIP) No 2016R1A2B4011045 and 2017R1C1B5015969.

\section{References}

1. Jung KW, Won YJ, Kong HJ, Oh CM, Cho H, Lee DH, et al. Cancer statistics in Korea: incidence, mortality, survival, and prevalence in 2012. Cancer Res Treat. 2015;47:127-41.

2. Torre LA, Bray F, Siegel RL, Ferlay J, Lortet-Tieulent J, Jemal A. Global cancer statistics, 2012. CA Cancer J Clin. 2015;65: 87-108.

3. Miller DL, Mayfield WR, Luu TD, Helms GA, Muster AR, Beckler VJ, et al. Community-based multidisciplinary computed tomography screening program improves lung cancer survival. Ann Thorac Surg. 2016;101:1864-9.

4. Patz EF Jr, Greco E, Gatsonis C, Pinsky P, Kramer BS, Aberle
DR. Lung cancer incidence and mortality in National Lung Screening Trial participants who underwent low-dose CT prevalence screening: a retrospective cohort analysis of a randomised, multicentre, diagnostic screening trial. Lancet Oncol. 2016;17:590-9.

5. Islami F, Torre LA, Jemal A. Global trends of lung cancer mortality and smoking prevalence. Transl Lung Cancer Res. 2015;4:327-38.

6. Choi MS, Park JS, Kim HK, Choi YS, Kim J, Shim YM, et al. Analysis of 1,067 cases of video-assisted thoracic surgery lobectomy. Korean J Thorac Cardiovasc Surg. 2011;44:169-77. 
7. Ikeda N, Saji H, Hagiwara M, Ohira T, Usuda J, Kajiwara N. Recent advances in video-assisted thoracoscopic surgery for lung cancer. Asian J Endosc Surg. 2013;6:9-13.

8. Salama JK, Vokes EE. New radiotherapy and chemoradiotherapy approaches for non-small-cell lung cancer. J Clin Oncol. 2013;31:1029-38.

9. Chang JY, Senan S, Paul MA, Mehran RJ, Louie AV, Balter P, et al. Stereotactic ablative radiotherapy versus lobectomy for operable stage I non-small-cell lung cancer: a pooled analysis of two randomised trials. Lancet Oncol. 2015;16:630-7.

10. Cheng TY, Cramb SM, Baade PD, Youlden DR, Nwogu C, Reid ME. The international epidemiology of lung cancer: latest trends, disparities, and tumor characteristics. J Thorac Oncol. 2016;11:1653-71.

11. Bray F, Jemal A, Grey N, Ferlay J, Forman D. Global cancer transitions according to the Human Development Index (20082030): a population-based study. Lancet Oncol. 2012;13:790801.

12. Jung KW, Won YJ, Oh CM, Kong HJ, Cho H, Lee JK, et al. Prediction of cancer incidence and mortality in Korea, 2016. Cancer Res Treat. 2016;48:451-7.

13. Lim D, Ha M, Song I. Trends in major cancer mortality in Korea, 1983-2012, with a joinpoint analysis. Cancer Epidemiol. 2015;39:939-46.

14. Gwon SH, Jeong S. Factors influencing adolescent lifetime smoking and current smoking in South Korea: using data from the 10th (2014) Korea Youth Risk Behavior Web-Based Survey. J Korean Acad Nurs. 2016;46:552-61.

15. Eckel SP, Cockburn M, Shu YH, Deng H, Lurmann FW, Liu L, et al. Air pollution affects lung cancer survival. Thorax. 2016;71:891-8.

16. Chen G, Wan X, Yang G, Zou X. Traffic-related air pollution and lung cancer: a meta-analysis. Thorac Cancer. 2015;6: 307-18.

17. Wang A, Qin F, Hedlin H, Desai M, Chlebowski R, Gomez S, et al. Physical activity and sedentary behavior in relation to lung cancer incidence and mortality in older women: The Women's Health Initiative. Int J Cancer. 2016;139:2178-92.

18. Koshy M, Fedewa SA, Malik R, Ferguson MK, Vigneswaran WT, Feldman L, et al. Improved survival associated with neoadjuvant chemoradiation in patients with clinical stage IIIA(N2) non-small-cell lung cancer. J Thorac Oncol. 2013;8: 915-22.

19. Kim HK, Cho JH, Choi YS, Zo JI, Shim YM, Park K, et al. Outcomes of neoadjuvant concurrent chemoradiotherapy followed by surgery for non-small-cell lung cancer with N2 disease. Lung Cancer. 2016;96:56-62.

20. Herskovic A, Chitti B, Christos P, Wernicke AG, Parashar B. Addition of surgery after radiation significantly improves survival in stage IIIB non-small cell lung cancer: a populationbased analysis. World J Surg. 2017;41:758-62.

21. Janet-Vendroux A, Loi M, Bobbio A, Lococo F, Lupo A, Ledinot $\mathrm{P}$, et al. Which is the role of pneumonectomy in the era of parenchymal-sparing procedures? Early / long-term survival and functional results of a single-center experience. Lung. 2015;193:965-73.

22. Park BJ, Flores RM, Rusch VW. Robotic assistance for videoassisted thoracic surgical lobectomy: technique and initial results. J Thorac Cardiovasc Surg. 2006;131:54-9.

23. Hirsch FR, Suda K, Wiens J, Bunn PA Jr. New and emerging targeted treatments in advanced non-small-cell lung cancer. Lancet. 2016;388:1012-24.

24. Shin A, Oh CM, Kim BW, Woo H, Won YJ, Lee JS. Lung cancer epidemiology in Korea. Cancer Res Treat. 2017;49:616-26. 